\title{
Desenvolvimento de uma Medida de Controle Primário e Secundário para Idosos
}

\author{
Hilma Tereza Tôrres Khoury ${ }^{1}$ \\ Universidade Federal do Pará \\ Isolda de Araújo Günther \\ Universidade de Brasília
}

\begin{abstract}
RESUMO - Apresenta-se neste artigo um instrumento desenvolvido para avaliar os processos adaptativos de controle primário e controle secundário em idosos. Controle primário é definido como uma estratégia utilizada para modificar o ambiente, visando adequá-lo às próprias necessidades. Controle secundário refere-se a esforços para adaptar-se ao ambiente. Participaram 315 idosos, entre 60 e 92 anos, sendo 33,3\% homens e 66,7\% mulheres. As entrevistas foram realizadas em seus domicílios. A análise fatorial identificou três fatores independentes: Esforço de Realização com Recursos Próprios (Controle Primário), Esforço de Adaptação (Controle Secundário) e Esforço de Realização com Ajuda (Controle Primário). Considerando a escassez de instrumentos disponíveis para avaliar esses construtos, espera-se que essa medida contribua para o avanço de pesquisas e serviços destinados aos idosos.
\end{abstract}

Palavras-chave: controle primário, controle secundário, idosos, controle percebido

\section{Development of a Measure of Primary and Secondary Control for Elderly People}

\begin{abstract}
This article presents a measure developed to evaluate the adaptive processes of primary and secondary controls in elderly persons. Primary control is defined as a strategy to modify the environment to attend to personal demands. Secondary control refers to efforts to fit in the environment. The participants were 315 elderly, between 60 and 92 years old, $33.3 \%$ males and $66.7 \%$ females. They were interviewed in their own homes. Using factor analysis, three independent factors were identified: Strive for Achievement with Own Resources (Primary Control), Adaptation Effort (Secondary Control) and Strive for Achievement with Help (Primary Control). Considering the paucity of instruments to evaluate these constructs, the measure should contribute to the advance of research as well as to services for elderly people.
\end{abstract}

Keywords: primary control, secondary control, elderly, perceived control

A população de pessoas com mais de 60 anos tem crescido em proporções nunca antes vista no Brasil, fato que tem mobilizado a sociedade e o governo no sentido de orientar o cuidado e a assistência à pessoa idosa, enfatizando o envelhecimento ativo. Dessa forma, a Política Nacional de Saúde da Pessoa Idosa - PNSPI (Ministério da Saúde, 2006) preconiza o envelhecimento saudável que implica manutenção da capacidade funcional - independência e autonomia - para por em prática as atividades de vida diária (AVDs). Isto significa que, apesar do desgaste biológico inerente à velhice, espera-se que o idoso seja capaz de produzir mudanças no seu ambiente, permanecendo independente e autônomo o máximo possível, em outras palavras, espera-se que o idoso seja capaz de exercer controle.

A manutenção da capacidade funcional depende não apenas do estado de saúde e das capacidades física e cognitiva, mas também de fatores psicossociais (Neri, 2006). Assim, por exemplo, não bastaria a um idoso ter condição de andar, saber fazer contas simples e conhecer a cidade para ir até o supermercado fazer suas compras. É preciso que ele acredite que possa realizar a tarefa e decida fazê-la. Inúmeros estu-

1 Endereço para correspondência: UFPA/IFCH/FAPSI Av. Augusto Corrêa, No. 1, Campus Básico, Guamá. Belém/PA. CEP: 66075-110.

E-mail:hilmatk@yahoo.com.br dos envolvendo população idosa atestaram associação entre percepção de controle e independência na velhice (Neri, 2006), qualidade de vida, bem estar ou satisfação com a vida (Haynes, Heckhausen, Chipperfield, Perry, \& Newall, 2009; Heckhausen, Wrosch, \& Fleeson, 2001; Heeps, 2000; Lang \& Heckhausen, 2001; Stocks, April, \& Lynton, 2012; Windsor, 2009; Wrosch, Heckhausen, \& Lachman, 2000) e saúde (Chipperfield et al., 2012; Chipperfield, Perry, Bailis, Ruthig, \& Loring, 2007; Chiu \& Spencer, 2010; Haynes et al., 2009; Wahl, Schilling, \& Becker, 2007; Widyarini, 2012; Windsor, 2009; Wrosch \& Heckhausen, 2002; Wrosch, Schulz, \& Heckhausen, 2002; Wrosch, Schulz, Miller, Lupien, \& Dunne, 2007). O conceito de controle assume diferentes denominações na literatura (como senso de controle, percepção de controle, lócus de controle, controle pessoal, controle primário e controle secundário) e faz parte de uma ampla área de estudos na psicologia, denominada de percepção de controle. Os conceitos de controle primário e controle secundário foram desenvolvidos por Rothbaum, Weisz e Snyder (1982) e integram o modelo de dois processos de controle a partir da crítica que fizeram aos modelos de processo único (Seligman, 1977). Esses construtos se referem ao nível ou grau de controle que as pessoas julgam ter sobre seu ambiente no sentido de modificá-lo ou adaptar-se a ele. $\mathrm{O}$ controle primário é definido como um processo que envolve 
“esforços para modificar o ambiente, de forma a adaptá-lo às necessidades do indivíduo" (Rothbaum et al., 1982, p. 8), ao passo que o secundário envolve "esforços para adaptar-se ao ambiente e fluir com a corrente "(p. 8).

Acreditar que está no controle da situação pode apoiar-se em fatos objetivos como as experiências de sucesso do indivíduo no sentido de alcançar suas metas e/ou conseguir apoio e reconhecimento social, mas, também, em fatores subjetivos tais como, por exemplo, acreditar na sorte e/ou na proteção de seres poderosos - reais ou imaginários. Sentir-se no controle é tão importante que, mesmo quando o indivíduo parece ter perdido o controle real da situação, encontra formas secundárias de resgatá-lo (Rothbaum et al., 1982). Exemplos disso são as reavaliações positivas na ocorrência de perdas ou fracassos e o rebaixamento dos níveis de aspiração quando as metas se tornam muito difíceis ou impossíveis de atingir. O esforço para modificar situações que oferecem resistência, característico do controle primário, tende a produzir alguma satisfação por sucessos, mas também algum desapontamento por falhas. Já o esforço para ajustar-se às resistências, característico do controle secundário, tende a ser experimentado como mais seguro e a evitar extremos de altos e baixos. Por essa razão, Rothbaum et al. (1982) sugeriram que a busca do controle secundário pode representar "um esforço para manter a crença na eventual efetividade do controle primário" (p. 9). Contudo, Rothbaum et al. (1982) e Weisz, Rothbaum e Blackburn (1984) afirmam que o mais importante é uma mistura adaptativa dos dois processos de controle.

Esses construtos foram utilizados pela Teoria do Controle no Curso de Vida (Heckhausen \& Schulz, 1995) para explicar o desenvolvimento humano ao longo da vida, sendo incorporados ao modelo Selection, Optimization and Compensation (SOC) de desenvolvimento bem-sucedido, traduzido como otimização seletiva com compensação (Baltes, 1997; Baltes \& Baltes, 1990). A Teoria do Controle no Curso de Vida e o modelo de desenvolvimento bem-sucedido que a integra (Schulz \& Heckhausen, 1996) propõem o construto de controle como tema central para a caracterização do desenvolvimento humano, da infância à velhice. Em seu ciclo de vida o indivíduo encontra oportunidades e restrições ao desenvolvimento de seu potencial para controle primário. Tais restrições são determinadas por duas grandes classes de fatores, os biológicos e os sociais. As mudanças biológicas universais, bem como os sistemas sociais com suas normas, determinariam o que um indivíduo pode fazer e quando pode fazê-lo, produzindo uma estrutura de oportunidades e de desafios à ampliação e manutenção do controle, ordenada pelo tempo. Durante a infância e a adolescência haveria um crescimento do potencial para controle primário. $\mathrm{O}$ controle primário permaneceria estável durante toda a vida adulta e velhice. Os adultos de várias idades percebem as mudanças na capacidade de controle primário ao longo da vida, mais especificamente, eles esperam declínios com o aumento da idade e particularmente na velhice avançada. Entretanto, embora a motivação para controle primário permaneça ativa, os indivíduos ajustam suas metas às mudanças de oportunidades. Em outras palavras, as pessoas tentam tirar o máximo de sua capacidade de controle em qualquer fase da vida (Heckhausen, Wrosch, \& Schulz, 2010). Na velhice, a manutenção do controle primário dependeria cada vez mais dos processos de controle secundário, particularmente o desengajamento de metas e a autoproteção. Portanto, a motivação para controle secundário cresceria com o avanço da idade (Heckhausen \& Schulz, 1995; Heckhausen et al., 2010; Schulz \& Heckhausen, 1996). A fim de lidar com as restrições ao seu desenvolvimento e de enfrentar os desafios a ele impostos de forma otimizada, o indivíduo precisaria "encontrar o equilíbrio entre controle primário e secundário" (Heckhausen \& Schulz, 1995, p. 290). O controle primário e o controle secundário são identificados tanto pela seleção quanto pela compensação. Estas proposições culminaram no chamado Modelo Optimization in Primary and Secondary Control (OPS), traduzido como otimização nos controles primário e secundário.

O modelo OPS prevê quatro tipos de estratégias de controle para a administração do curso de vida individual: controle primário seletivo, controle primário compensatório, controle secundário seletivo e controle secundário compensatório. $\mathrm{O}$ indivíduo teria de ativar essas estratégias e mantê-las em equilíbrio, conforme as demandas específicas encontradas em um dado período do ciclo de vida, de forma a otimizar seu desenvolvimento. As quatro estratégias de controle foram assim definidas (Heckhausen, 1997; Heckhausen, Schulz, \& Wrosch, 1998; Schulz \& Heckhausen, 1996):

1) Controle primário seletivo: refere-se ao investimento focalizado de recursos tais como esforço, tempo, capacidades e habilidades requeridos para o alcance de uma meta escolhida. "É ação que visa diretamente atingir metas" (Heckhausen, 1997, p. 177). Por exemplo, o desenvolvimento de capacidades e habilidades pelos processos de aquisição e prática.

2) Controle primário compensatório: é requerido quando os recursos internos do indivíduo (capacidades físicas ou cognitivas) são insuficientes para atingir uma meta escolhida, tal como ocorre com crianças pequenas ou frágeis ou idosos. Refere-se ao uso de recursos externos ao indivíduo, tais como a ajuda de outras pessoas ou auxílios técnicos (cadeira de rodas, andador, aparelho de surdez e assemelhados) para complementar as próprias capacidades. Inclui também o emprego de meios novos e incomuns para substituir habilidades perdidas ou indisponíveis, como a leitura labial para deficientes auditivos.

3) Controle secundário seletivo: refere-se às "representações internas" (Schulz \& Heckhausen, 1996, p. 710) relevantes do ponto de vista da motivação para a persecução de uma meta escolhida. "É dirigido ao mundo interno do indivíduo para promover o compromisso da vontade para com uma meta escolhida" (Heckhausen, 1997, p. 177), tal como valorizar uma meta escolhida ou aumentar o controle pessoal percebido em relação ao alcance da meta.

4) Controle secundário compensatório: refere-se a estratégias que têm como finalidade amortecer ou minimizar os efeitos negativos de falhas ou perdas sobre a autoestima do indivíduo e seus recursos motivacionais (por exemplo, crença de autoeficácia) 
para o controle primário. Exemplos: desengajamento de metas, atribuição causal autoprotetora, comparações estratégicas - intraindividual e social.

$\mathrm{Na}$ velhice, o natural desgaste biológico e as mudanças de papéis sociais impõem limites ao exercício do controle. Estudos empíricos demonstraram que a percepção de controle sofre modificações com o avanço da idade (Heckhausen, 1997; Heckhausen et al., 2010; Lang \& Heckhausen, 2001; Wrosch \& Heckhausen, 2002; Wrosch et al., 2000), havendo um redirecionamento do controle primário para metas apropriadas à idade (Heckhausen, 1997; Heckhausen et al., 2001, 2010; Wrosch \& Heckhausen, 1999) e uma tendência a buscar controle secundário (Heckhausen, 1997; Heckhausen et al., 2010; Peng \& Lachman, 1994). Assim, recursos pessoais como experiências passadas de sucesso, comparação social, flexibilidade no ajustamento de metas e reavaliações positivas, por exemplo, se tornam muito importantes no sentido de manter o senso de estar no comando (Heckhausen et al., 2010; Schulz \& Heckhausen, 1996).

À época em que a presente pesquisa foi realizada (2003 e 2004), os estudos que haviam avaliado controle primário e secundário em idosos eram todos em língua inglesa, produzidos fora do Brasil. Alguns tinham utilizado medidas com número reduzido de itens (Lang \& Heckhausen, 2001; McConatha \& Huba, 1999; Wrosch \& Heckhausen, 2002; Wrosch et al., 2000, 2002) ou voltadas para estudar esses processos em domínios específicos como saúde (Heckhausen et al., 2001; Wrosch et al., 2002). Poucos estudos disponibilizavam medidas abordando os processos de controle de forma geral (sem focalizar em domínios específicos), contendo pelo menos 20 itens, e apresentando boa consistência. Dentre estes, dois utilizaram medidas de controle primário e secundário (Heckhausen et al., 1998; Peng \& Lachmann, 1994) e um empregou medida relacionada a esses construtos (Brandtstädter \& Renner, 1990). Esse panorama pouco mudou desde então. Estudos recentes (Chipperfield et al., 2007; Haynes et al., 2009; Wahl et al., 2007; Widyarini, 2012) investigaram controle primário e controle secundário acessando-os por meio dos instrumentos de medida existentes àquela época, utilizando-os na íntegra, em parte, ou com adaptações dos itens originais para avaliar situações específicas. Assim, considerou-se importante desenvolver um instrumento para avaliar o quanto de controle um idoso acredita ter sobre seu ambiente e sobre si, de forma a orientar intervenções psicossociais no sentido de estimular a manutenção da capacidade funcional na velhice.

Para elaboração de seu instrumento, Brandtstädter e Renner (1990) partiram da perspectiva de curso de vida (life-span) que aborda os ganhos e as perdas ao longo do desenvolvimento humano e a consequente necessidade de maximizar ganhos e minimizar perdas (Baltes \& Baltes, 1990). O instrumento proposto por Brandtstädter e Renner (1990) possui dois fatores independentes - Tenacious Goal Pursuit e Flexible Goal Adjustment - relativos a modos distintos, porém, complementares de enfrentamento de crises e transições críticas da vida, denominados de tendência assimilativa e tendência acomodativa. A primeira diz respeito à transformação das circunstâncias de desenvolvimento pessoal de acordo com as próprias preferências (ex.: Quando confrontado com obstáculos, costumo dobrar meus esforços). A segunda se refere ao ajustamento das preferências pessoais às restrições situacionais. (ex.: Eu penso que mesmo os problemas da vida têm seu lado bom). Essas duas dimensões parecem corresponder ao controle primário e secundário na definição original (Rothbaum et al., 1982; Weisz et al., 1984).

Peng e Lachmann (1994) elaboraram um instrumento para medir controle primário e controle secundário com base nas definições de Rothbaum et al. (1982) e avançando a partir do instrumento de Brandtstädter e Renner (1990) que teriam enfocado processos de controle envolvendo apenas o indivíduo. Peng e Lachmann (1994) propuseram processos de controle primário e secundário sensíveis a aspectos sociais (ex.: Eu tento fazer com que os outros vejam o meu ponto de vista em uma disputa/Eu costumo resolver uma disputa concordando com o ponto de vista da outra pessoa) e do envelhecimento (lutar contra o envelhecimento versus aceitar o envelhecimento), bem como orientação a metas (ex.: Quando me defronto com uma situação ruim, eu faço qualquer coisa para torná-la melhor/Quando me defronto com uma situação ruim, eu me sinto melhor modificando a minha maneira de ver as coisas). Peng e Lachmann (1994) compararam dois grupos culturalmente distintos. Em ambos os grupos ocorreram correlações positivas entre o controle primário e a dimensão Tenacious Goal Pursuit de Brandtstädter e Renner (1990), bem como entre o controle secundário e a dimensão Flexible Goal Adjustment. O instrumento desenvolvido por Peng e Lachman serviu de base para acessar controle primário e secundário em diversos estudos (Chipperfield et al., 2007; Haynes et al., 2009; Wrosch et al., 2000).

Heckhausen et al. (1998) elaboraram uma escala de acordo com o Modelo OPS (Heckhausen \& Schulz, 1995; Schulz \& Heckhausen, 1996), composta por quatro fatores de controle e mais um, denominado de otimização. $O$ fator controle primário seletivo congrega itens abordando investimento em esforço e tempo, desenvolvimento de habilidades e luta contra dificuldade para atingir metas (ex.: Quando eu realmente quero alguma coisa, sou capaz de trabalhar duro para alcançá-la). O fator controle primário compensatório reúne itens cujo conteúdo versa sobre ajuda ou conselho de outras pessoas, habilidades externas para atividade tais como meios novos e incomuns e fazer um desvio (ex.: Quando obstáculos surgem em meu caminho, eu tento obter ajuda de outras pessoas/Quando obstáculos surgem em meu caminho, eu encontro outra maneira de conseguir o que eu quero). A dimensão controle secundário seletivo engloba itens que tratam de aumento do valor da meta e da percepção de controle para com as metas escolhidas, desvalorização de outras metas e antecipação de consequências positivas que adviriam do alcance da meta (ex:. Quando tomo uma decisão sobre alguma coisa, eu sempre me lembro de que foi a decisão certa/Para metas que são difíceis de atingir, eu penso no quão orgulhoso eu me sentirei quando as tiver alcançado). A dimensão controle secundário compensatório abarca itens que envolvem o desengajamento de metas, atribuição autoprotetora, e comparação social e intraindividual (ex.: Quando uma coisa se torna muito difícil, eu a tiro de meus pensamentos). O fator otimização aborda aspectos tais como investimento de longo prazo em metas 
potenciais, mudança de metas para adaptar-se a tarefas do desenvolvimento, manutenção de múltiplos domínios de metas (ex.: Muitas metas de vida se tornam importantes para mim porque é a hora certa para elas/Eu permaneço ativo e envolvido em muitos e diferentes setores da vida). A escala OPS mostrou correlação positiva com indicadores de bem estar psicológico e foi utilizada para acessar controle primário e secundário em vários estudos (Chipperfield et al., 2007; Haynes et al., 2009; Wahl et al., 2007; Widyarini, 2012; Wrosch \& Heckhausen, 1999; Wrosch et al., 2002), tendo sido utilizada na totalidade, em parte, ou com adaptações.

Considerando que o Modelo OPS havia avançado a partir do conceito originalmente proposto por Rothbaum et al. (1982) e, além disso, integrava o modelo SOC (Baltes, 1997; Baltes \& Baltes, 1990), decidiu-se utilizar o instrumento de Heckhausen et al. (1998) para o estudo de controle primário e secundário em idosos. Foi realizada sua tradução para a língua portuguesa, efetuada pelas autoras, com posterior tradução inversa realizada por psicólogos bilíngues. Contudo, a partir de estudos piloto, alguns itens não se mostraram adequados para seu uso no Brasil, já que as formas de obtenção dos dois tipos de controle são influenciadas pelo contexto cultural. Assim sendo, decidiu-se desenvolver e validar um instrumento para medir a percepção de controle primário e controle secundário em idosos, tendo a escala OPS como base, porém com itens adaptados à realidade brasileira, constituindo-se este o objetivo do estudo.

\section{Método}

\section{Participantes}

Por meio de amostragem não-probabilística, por quotas de gênero, participaram neste estudo 315 indivíduos, sendo $33,3 \%$ homens e $66,7 \%$ mulheres, residentes em Brasília-DF, com idades entre 60 e 92 anos $(M=71,3 ; D P=6,9)$. A população com 60 anos e acima na cidade pesquisada, conforme o Censo 2000 do IBGE, era composta por 7.897 homens e 11.066 mulheres, representando respectivamente $41,6 \%$ e $58,4 \%$ do total da população de 18.963 indivíduos. Como a escala submetida à validação foi composta por 28 itens, aplicados a 315 sujeitos, a proporção foi de 11,25 sujeitos por item, atendendo, portanto, aos critérios psicométricos exigidos para validação de instrumentos (Pasquali, 2010).

Os participantes tinham, em média, 11,1 anos de estudo (mínimo 6 meses; máximo 25 anos; $M d n=12,0 ; D P=5,5$ ), renda familiar mensal de $\mathrm{R} \$$ 5.043,64 (mínimo $\mathrm{R} \$ 240,00$; máximo $\mathrm{R} \$ 20.000,00 ; M d n=\mathrm{R} \$ 4.500,00 ; D P=3227,09)$ e renda familiar mensal per capita de R\$2.077,93 (mínimo $\mathrm{R} \$ 111,11$; máximo $\mathrm{R} \$ 14.000,00 ; M d n=\mathrm{R} \$ 1.500,00 ; D P=$ $1849,98)$. Entre os participantes, 14,9\% moravam sozinhos, 46\% não tinham companheiro, sendo que destes, $73,8 \%$ eram viúvos. Mais de dois terços $(67,3 \%)$ eram aposentados, $61,3 \%$ contribuíam integralmente ou com a maior parte dos recursos para o sustento da família; $18,4 \%$ ainda trabalhavam. A maioria (88,6\%) declarou sair sozinha, mesmo que fosse apenas para lugares próximos à residência; 76,8\% saíam de casa diariamente; $40 \%$ dirigiam o próprio automóvel; $34,9 \%$ andavam de ônibus.

\section{Instrumento}

No processo de construção do instrumento para medir a percepção de controle primário e secundário em idosos (ECOPSE), adotou-se a estrutura de quatro fatores de controle, de acordo com Heckhausen et al. (1998). Os itens foram elaborados com base em Peng e Lachmann (1994), Brandstädter e Renner (1990) e Heckhausen et al. (1998), adaptando-os às particularidades culturais e sociais do Brasil. A construção dos itens seguiu as recomendações para construção de instrumentos de medida referentes a construto (Pasquali, 2010).

A escala foi inicialmente estruturada com 32 itens, ficando reduzida a 28 após a validação semântica. Os itens foram distribuídos em 4 subescalas: Controle Primário Seletivo, Controle Primário Compensatório, Controle Secundário Seletivo e Controle Secundário Compensatório (Heckhausen et al., 1998). Em cada subescala os itens correspondiam a determinados aspectos, tudo em conformidade com o modelo OPS.

Os aspectos avaliados em cada subescala foram os seguintes: a) Controle Primário Seletivo - investimento em esforço, investimento em tempo, desenvolvimento de habilidades e capacidades relevantes; b) Controle Primário Compensatório - busca de assistência de outras pessoas para tarefas motoras, busca de assistência de outras pessoas para tarefas cognitivas, e utilização de auxílio técnico; c) Controle Secundário Seletivo - elevação do valor da meta escolhida, desvalorização de metas não escolhidas, aumento da percepção de controle para o alcance de metas, antecipação de consequências positivas relacionadas ao alcance de metas e ajustamento do nível de aspiração para alcançar uma meta; e d) Controle Secundário Compensatório: desengajamento de metas anteriores não alcançadas, atribuição autoprotetora, comparação social, comparação intraindividual, rebaixamento do nível de aspiração frente a não realização.

No intuito de aperfeiçoar os itens, tornando-os mais compreensíveis aos estratos menos escolarizados da população alvo, realizou-se a validação semântica dos mesmos, efetuada em duas etapas, antes e durante o último estudo piloto. A primeira etapa da validação semântica foi realizada com 10 participantes, homens e mulheres com diferentes graus de instrução. Na segunda etapa, realizada com trinta participantes, alguns itens ainda sofreram modificação, particularmente substituição de palavras por sinônimos (Khoury, 2005).

Assim, a versão submetida à análise fatorial foi composta por 28 itens, avaliados em escala do tipo Likert com cinco pontos $(0=$ nada a ver comigo; $1=$ pouco a ver comigo; $2=$ mais ou menos a ver comigo; 3 = muito a ver comigo; $4=$ tudo a ver comigo). Procurou-se assegurar que cada aspecto componente de um fator tivesse pelo menos dois itens. Tal medida foi tomada visando à posterior análise fatorial, na qual alguns ou vários itens poderiam desaparecer em função do tamanho de sua carga fatorial e do coeficiente alfa do fator ao qual pertencessem. 


\section{Procedimentos}

Os participantes foram entrevistados em suas residências, após assinatura do Termo de Consentimento Livre e Esclarecido. A amostra foi recrutada em diferentes áreas da cidade, que foram percorridas em busca dos potenciais participantes. Contudo, devido à grande dificuldade para entrar nos domicílios e realizar as entrevistas (tentativas anteriores de coleta de dados via questionários autoadministrados tinham se mostrado ineficientes com população idosa), parte dos dados foram coletados por meio da técnica bola de neve. Quando se conseguia entrar em um apartamento e entrevistar alguém, solicitava-se ao entrevistado que indicasse outras pessoas idosas naquela área. Entretanto, quando já se tinha uma boa rede de entrevistados gerada por um dos participantes, mudava-se de área, a fim de evitar tendenciosidade. A aplicação da escala de controle primário e controle secundário, denominada de ECOPSE, teve duração média de 20 minutos.

\section{Análise de Dados}

A fatorabilidade da matriz de correlações foi decidida pelo teste de adequação da amostra de Kaiser-Meyer-Olkin (KMO). A correlação entre as variáveis foi verificada pelo Teste de Esfericidade de Bartlett. A estrutura fatorial da matriz de intercorrelações foi investigada pela Análise de Componentes Principais. Foram utilizados dois critérios para identificar o número de fatores a serem extraídos: a análise do diagrama de declividade (screeplot), adotando-se como ponto de corte o ponto de inflexão da curva (Cattell citado por Field, 2009) e a análise da porcentagem de variância explicada, obtida a partir do acréscimo de cada fator. Stevens (citado por Field, 2009) argumenta que o diagrama de declividade fornece um critério bastante confiável para a seleção dos fatores com amostras de mais de 200 participantes. Contudo, afirma Field (2009), a seleção de fatores não deve ser baseada em um único critério.

A análise fatorial foi processada por meio do método de extração dos eixos principais (PAF) e rotação oblíqua baseada no pattern (direct oblimin). A opção por esse tipo de rotação está fundamentada no seguinte:

a) Ela permite que apareçam fatores ortogonais quando estes existem, já que não força a dependência entre os fatores, apenas a permite, enquanto a rotação ortogonal só permite fatores independentes. Considerando-se que os traços latentes em Psicologia quase nunca são independentes, a rotação oblíqua respeita este aspecto da correlação entre os fatores (Pasquali, 2005).

b) A teoria que embasou a construção do instrumento ora sob validação, o modelo OPS e sua correspondente Teoria do Controle no Curso de Vida (Heckhausen \& Schulz, 1995; Schulz \& Heckhausen, 1996), propõe duas dimensões como estratégias de controle pessoal, portanto, faces de uma mesma moeda: o controle primário e o controle secundário. A Teoria do Controle no Curso de Vida (Heckhausen \& Schulz, 1995) argumenta que, na velhice, o controle secundário tem a função de conservar o potencial para o controle primário.
Para a decisão das variáveis componentes de cada fator foi solicitado que as matrizes mostrassem somente as cargas fatoriais a partir de 0,40 , índice de validade do item considerado próximo ao razoável na classificação de Comrey (citado por Pasquali, 2005). Foi realizada também a análise da consistência dos fatores, por meio do coeficiente alfa de Cronbach (critério mínimo de consistência $=0,70$ ).

\section{Resultados}

Objetivou-se com este estudo desenvolver um instrumento para medir a percepção de controle primário e controle secundário em idosos, denominado de ECOPSE. A validade de construto foi analisada visando estabelecer sua estrutura fatorial. Esperava-se que a estrutura fatorial confirmasse os quatro componentes propostos pela teoria do controle no curso de vida e seu correspondente modelo OPS (Heckhausen \& Schulz, 1995; Schulz \& Heckhausen, 1996).

Por meio da Análise dos Componentes Principais obteve-se um KMO igual a 0,8, valor considerado bom na classificação de Kaiser (citado por Pasquali, 2005), indicando que a matriz de correlações é passível de fatorização. Outro indício da fatorabilidade da matriz de correlações obtida com os dados desta pesquisa é que o determinante da matriz é zero, significando que seu posto - número mínimo de fatores a serem inicialmente extraídos - é menor que o número de variáveis. A correlação entre as variáveis foi verificada pelo Teste de Esfericidade de Bartlett $(p=0,001)$. O diagrama de declividade (screeplot) apontava três fatores a serem extraídos com segurança, observando-se o ponto de inflexão da curva. Contudo, considerando-se que a fundamentação teórica para a construção do instrumento previa quatro fatores de controle, foi realizada primeiramente uma solução com quatro fatores. Nessa solução, oito itens foram perdidos $(11,6,22,9,4,7,18$, e 13) por apresentarem carga fatorial abaixo de 0,40 . Além disso, o quarto fator ficou com apenas dois itens caracterizados como controle secundário compensatório. Todos os outros itens qualificados como controle secundário compensatório haviam se agrupado no Fator 2. O Fator 1 reuniu tanto os itens caracterizados como controle primário seletivo, quanto aqueles tidos como controle secundário seletivo, exceto dois que ficaram no Fator 2. O Fator 3 agrupou os itens qualificados como controle primário compensatório. Diante deste resultado, e levando-se em conta que a análise da porcentagem de variância explicada obtida a partir do acréscimo de cada fator mostrou que quatro fatores acrescentariam apenas $5,5 \%$ à explicação da variância, decidiu-se proceder à solução com três fatores (ver Tabela 1).

Na solução com três fatores, igualmente oito itens foram excluídos $(6,11,13,22,4,2,7$ e 18) por apresentarem carga fatorial abaixo de 0,40 . Observa-se que os itens excluídos nas duas soluções são os mesmos, exceto os itens 9 (excluído na solução com quatro fatores) e o item 2 (excluído na solução com três fatores). Os itens restantes foram submetidos à análise de consistência interna por meio do cálculo do coeficiente alfa de Cronbach. Na solução com três fatores, o Fator 1 agregou os itens caracterizados como controle primário seletivo e a maioria dos itens qualificados como controle 
Tabela 1. Matriz Padrão da ECOPSE com Três Fatores

\begin{tabular}{|c|c|c|c|c|}
\hline \multirow{2}{*}{\multicolumn{2}{|c|}{ Itens Conteúdo }} & \multicolumn{3}{|c|}{ Cargas Fatoriais } \\
\hline & & \multirow{2}{*}{$\frac{\mathrm{F} 1}{0,695}$} & \multirow[t]{2}{*}{$\mathrm{F} 2$} & \multirow[t]{2}{*}{ F3 } \\
\hline 24 & Quando tenho um objetivo, procuro pensar que sou capaz de alcançar & & & \\
\hline 14 & Quando realmente quero alguma coisa, faço todo o possível para conseguir & 0,672 & & \\
\hline 12 & Quando planejo fazer alguma coisa, acredito firmemente que vou conseguir realizar & 0,595 & & \\
\hline 17 & Diante das dificuldades, eu penso que, no final de tudo, a luta vai valer a pena! & 0,512 & & \\
\hline 16 & Quando decido fazer alguma coisa, procuro praticar bastante para fazer cada vez melhor. & 0,503 & & \\
\hline 8 & Quando alguma coisa é importante para mim, dedico todo o tempo que posso a ela & 0,501 & & \\
\hline 20 & Quando quero muito alguma coisa, eu dou prioridade a ela (coloco em primeiro lugar) & 0,485 & & \\
\hline 1 & Quando tenho um sonho (aspiração), me esforço para transformar em realidade (para concretizar) & 0,456 & & \\
\hline 5 & Quando decido fazer alguma coisa, procuro aprender tudo o que for preciso para fazer bem feito & 0,423 & & \\
\hline 27 & Se o que quero fica difícil de alcançar, procuro uma coisa mais modesta & & 0,627 & \\
\hline 21 & Quando o que quero está difícil de conseguir, passo a querer algo mais fácil (de conseguir) & & 0,588 & \\
\hline 19 & Quando planejo uma coisa e não consigo realizar, penso: "Vai ver sonhei alto demais"! & & 0,536 & \\
\hline 25 & Quando não consigo o que quero, admito que meus objetivos estavam fora da realidade & & 0,527 & \\
\hline 26 & $\begin{array}{l}\text { Quando falho em alguma coisa, me consolo, lembrando de outras coisas onde já fui mais bem } \\
\text { sucedido }\end{array}$ & & 0,482 & \\
\hline 9 & Quando o que quero fica muito difícil de alcançar, penso: "Vai ver não era para ser" & & 0,462 & \\
\hline 15 & Se uma coisa que quero se torna muito difícil de alcançar, eu mudo (troco) para outra coisa & & 0,409 & \\
\hline 10 & Quando não consigo fazer algo sozinho, peço ajuda a alguém & & & $-0,762$ \\
\hline 3 & Quando tenho dificuldade para fazer alguma coisa, busco a ajuda de outras pessoas & & & $-0,680$ \\
\hline 28 & $\begin{array}{l}\text { Quando me enrolo para resolver um problema, consulto outras pessoas (me aconselho com } \\
\text { outras pessoas) }\end{array}$ & & & $-0,524$ \\
\hline \multirow[t]{3}{*}{23} & Quando não consigo compreender alguma coisa, pergunto a quem sabe & & & $-0,489$ \\
\hline & Alfa de Cronbach & 0,79 & 0,74 & 0,76 \\
\hline & Número de Itens & 9 & 7 & 4 \\
\hline
\end{tabular}

secundário seletivo, exceto dois (27 e 21) que se juntaram ao Fator 2. Por sua vez, o Fator 2 reuniu os itens caracterizados como controle secundário compensatório, exceto os dois já mencionados. O conteúdo destes itens ( 27 e 21 ) diz respeito ao rebaixamento do nível de aspiração, portanto, um consolo em caso de metas não alcançadas ou difíceis de alcançar. O Fator 3 congregou os itens qualificados como controle primário compensatório (ver Tabela 1).

Assim, a estrutura fatorial da ECOPSE apresenta três fatores independentes com boa consistência interna e que juntos explicam 36,5\% da variância total do construto: a) Esforço de Realização com os Próprios Recursos - Controle Primário (nove itens; $\alpha=0,79$ ) que diz respeito a esforços de realização dirigidos à concretização de metas; por meio dos recursos pessoais do indivíduo; b) Esforço de Adaptação - Controle Secundário (sete itens; $\alpha=0,74$ ) que se refere a esforços no sentido de compensar perdas/falhas ou consolar-se em caso de metas não alcançadas ou difíceis de alcançar; c) Esforço de Realização com Ajuda - Controle Primário (quatro itens; $\alpha=0,76$ ), concernente a esforços de realização dirigidos à concretização de metas, porém, com ajuda de outras pessoas.

\section{Discussão}

A elaboração da ECOPSE fundamentou-se nos conceitos preconizados pela teoria do controle no curso de vida e seu correspondente modelo OPS (Heckhausen \& Schulz, 1995; Schulz \& Heckhausen, 1996). Contudo, diferentemente do modelo bidimensional de seleção/compensação e de controle primário/secundário (modelo OPS), que postula quatro dimensões de controle (controle primário seletivo, controle primário compensatório, controle secundário seletivo, controle secundário compensatório), o resultado da análise fatorial identificou somente três fatores. Para representar esses fatores, foram mantidos 20 dos 28 itens submetidos à análise, os quais se mostraram representativos dos fatores aos quais pertencem.

No Fator 1, Esforço de realização com recursos próprios, os itens expressam o esforço do indivíduo para alcançar metas, seja no sentido de empenhar-se concretamente para isto, seja no sentido de se automotivar para tentar alcançá-las. O conteúdo deste fator corresponde ao que na teoria do controle no curso de vida se caracterizaria como controle seletivo, englobando tanto o primário seletivo quanto o secundário seletivo (Heckhausen \& Schulz, 1995; Schulz \& Heckhausen, 
1996; Heckhausen, 1997). Os itens submetidos à análise, e que representavam a dimensão controle secundário seletivo, se agruparam com os itens que representavam a dimensão controle primário seletivo e compuseram um único fator, cujo conteúdo diz respeito a esforço de realização com as próprias capacidades ou recursos. Neste sentido, a estratégia denominada de controle secundário seletivo, tal como apresentada na teoria do controle no curso de vida e no modelo OPS, não foi confirmada neste estudo como uma dimensão de controle secundário.

Uma possível explicação para esse resultado é que, de acordo com o modelo das fases da ação na regulação do desenvolvimento, que faz parte da Teoria do Controle no Curso de Vida (Heckhausen \& Schulz, 1995; Heckhausen et al., 2010), a função do controle secundário seletivo consiste em aumentar o comprometimento do sujeito para com metas escolhidas e mantê-lo focalizado nestas metas, a fim de atingi-las. $\mathrm{O}$ controle secundário seletivo tem função motivacional no sentido de dirigir a ação do sujeito para alcançar uma meta selecionada. Portanto, em última instância esta estratégia visa ao controle primário, faz parte do processo para submeter o ambiente à própria vontade $\mathrm{e}$ obter controle primário (Khoury \& Günther, 2009). Assim sendo, o conteúdo dos itens da ECOPSE que originalmente integraram a dimensão controle secundário seletivo seria mais adequadamente classificado como controle primário, conforme mostra a análise fatorial quando agrega os itens desta dimensão aos da dimensão controle primário seletivo. O esforço de realização evidenciado neste estudo está voltado para o controle primário, seja no sentido da ação efetiva do indivíduo, seja no sentido de sua motivação para agir ou enfrentar dificuldades, de forma a atingir seus objetivos, o que estaria de acordo com a concepção original de controle primário (Rothbaum et al., 1982; Weisz et al., 1984), bem como com o aperfeiçoamento posterior deste conceito empreendido por Thurber e Weisz (1997), os quais distinguiram entre métodos e metas de enfrentamento. Os métodos são maneiras de agir e de pensar e são classificados como comportamentos observáveis e não observáveis. As metas são os fins aos quais os métodos de enfrentamento se dirigem. Estes autores afirmaram que "os conceitos de controle primário e secundário se aplicam apenas às metas de enfrentamento porque somente metas têm um objeto de controle, seja o self (controle secundário) ou as condições objetivas (controle primário)" (Thurber \& Weisz, 1997, p. 509). Assim, se a meta é mudar o próprio indivíduo para que se adapte às circunstâncias, isto significa controle secundário, por outro lado, se a meta é modificar o ambiente para satisfazer as necessidades do indivíduo, isto é controle primário, não importando, em ambos os casos, se o meio utilizado é comportamental ou cognitivo.

No Fator 2, Esforço de Adaptação, verifica-se que todos os itens expressam a busca de alguma forma de compensação ou de consolo quando o objetivo está difícil de ser atingido ou não é alcançado ou, ainda, em caso de falha, fracasso ou adversidade. Segundo a Teoria do Controle no Curso de Vida, estes itens caracterizariam o controle secundário compensatório e correspondem ao controle secundário na formulação original (Rothbaum et al., 1982; Weisz et al., 1984), bem como ao Fator Tendência Acomodativa de Brandtstädter e Renner (1990). A exceção do controle secundário compensatório, todas as outras estratégias de controle propostas por Heckhausen e Schulz (1995), até mesmo a estratégia denominada de controle secundário seletivo, visam ao alcance de metas, portanto, ao controle primário.

No Fator 3, todos os itens se referem ao esforço de realização por meio da ajuda de outras pessoas, em caso de dificuldade ou necessidade, de tal forma que o sujeito possa alcançar seus objetivos. O conteúdo deste fator representa o que na Teoria do Controle no Curso de Vida se caracterizaria como controle primário compensatório (Heckhausen, 1997; Heckhausen \& Schulz, 1995; Schulz \& Heckhausen, 1996). Essa distinção do controle primário nas categorias seletivo e compensatório parece apropriada ao estudo do desenvolvimento humano, especialmente considerando-se que em todas as fases da vida, mormente na infância e na velhice, o ser humano necessita de ajuda externa para concretizar muitos de seus objetivos. Porém, a utilização do termo compensatório exige cautela, uma vez que, neste caso, o sujeito está buscando recursos que lhe auxiliem a levar a cabo sua seleção, ou seja, a atingir suas metas (Khoury \& Günther, 2009).

A estrutura fatorial encontrada se coaduna com a definição original de controle primário e secundário (Rothbaum et al., 1982), bem como com o desenvolvimento posterior desses conceitos (Thurber \& Weisz, 1997) que propõem o controle primário como esforços para dominar o ambiente e adaptá-lo às próprias necessidades, e o controle secundário como esforços para se adaptar ao ambiente ou circunstâncias. A meta do controle primário é o ambiente externo ao indivíduo, enquanto que a meta do controle secundário é o indivíduo. Contudo, os meios ou métodos utilizados pela pessoa para conseguir controle primário ou secundário podem ser comportamentais ou cognitivos. Os resultados obtidos oferecem apoio aos estudos de Peng e Lachman (1994) e Heeps (2000), cujos instrumentos para medir controle primário e secundário tiveram como fundamento as definições de Rothbaum et al. (1982), bem como ao de Brandtstädter e Renner (1990), cuja tendência assimilativa diz respeito à transformação das circunstâncias de desenvolvimento de acordo com as preferências pessoais e a tendência acomodativa, ao ajustamento das preferências pessoais às restrições situacionais. Por outro lado, os resultados contrariaram a estrutura de quatro fatores de controle proposta pelo modelo OPS (Heckhausen \& Schulz, 1995; Schulz \& Heckhausen, 1996). Embora a teoria do controle no curso de vida e o modelo OPS que lhe corresponde sejam fecundos na abordagem teórica dos processos adaptativos de controle primário e secundário, analisando-se as quatro estratégias de controle propostas, verifica-se que, a exceção do controle secundário compensatório, todas as outras visam ao alcance de metas, por conseguinte, têm em vista o controle primário. A estratégia denominada de controle secundário seletivo refere-se ao comprometimento motivacional do sujeito para com as metas selecionadas, portanto, um meio para modificar o ambiente externo na direção pretendida. Desse modo, é compreensível que os itens que diziam respeito a essa estratégia na versão original da ECOPSE tenham se agregado aos da dimensão controle primário seletivo, formando um único fator. 
Outra explicação para esse resultado advém do fato de que muitos dos estudos empíricos que utilizaram a escala OPS (Heckhausen et al., 1998) como medida dos construtos de controle primário e secundário não a empregaram em sua totalidade, tendo utilizado apenas algumas de suas subescalas ou alguns de seus itens (Chipperfield et al., 2007; Wrosch et al., 2002; Haynes et al., 2009; Widyarini, 2012), adaptados para estudar situações específicas. Isto sugere carência de confirmação empírica para a estrutura de quatro fatores e aponta para a necessidade de mais investigações no sentido de testar a estrutura proposta pelo modelo OPS.

O resultado do presente estudo corroborou o controle primário como um esforço do indivíduo para a concretização de suas metas. O empenho concreto dirigido à realização pode ser empreendido por meio de recursos pessoais (do indivíduo) ou de ajuda externa (de outras pessoas ou de tecnologias). Os recursos pessoais incluem a automotivação para o alcance das metas e as intervenções no ambiente com esse fim, exercidas com os recursos individuais. $\mathrm{O}$ controle secundário confirmou-se como um esforço de adaptação, no sentido de acomodação ou adequação a situações em que metas não foram alcançadas ou se tornaram difíceis de atingir, ou seja, situações onde o controle primário não foi possível, de tal forma que o evento incontrolável possa ser percebido como controlável em um sentido secundário, uma segunda via (Rothbaum et al., 1982). O resultado da análise fatorial aqui processada permite concluir que o controle primário apresenta duas facetas, mas o controle secundário, apenas uma.

A ECOPSE tem como quadro de referência o modelo teórico que conceitua percepção de controle como um processo adaptativo cujo objetivo consiste em alcançar a meta desejada ou, em caso de perda/falha, adaptar-se às circunstâncias. $\mathrm{O}$ instrumento se mostrou adequado do ponto de vista psicométrico. Recomenda-se sua utilização na pesquisa e na prática clínica. Na pesquisa, os processos de controle podem ser utilizados para investigar sua relação com capacidade funcional, comportamentos de saúde e qualidade de vida, por exemplo. Na prática clínica, seu emprego pode identificar fatores psicossociais (percepção de controle) que favorecem ou dificultam comportamentos de saúde tal como adesão ao tratamento e capacidade funcional (critério de velhice saudável), contribuindo para orientar intervenções psicológicas que promovam saúde e qualidade de vida.

$\mathrm{O}$ fato de o presente estudo ter sido realizado com amostra não-probabilística e em apenas uma cidade constitui-se em uma limitação que pode dificultar a sua generalização para outras populações de idosos no país. Assim, sugere-se que estudos semelhantes sejam realizados em outras cidades, em diferentes regiões do país, se possível com amostras aleatórias, a fim de testar a estrutura encontrada. Além disso, como os itens não são específicos para idosos, sugere-se que o instrumento possa ser utilizado com outras populações, como adultos e jovens, buscando não apenas testar sua estrutura fatorial, mas também estudar os processos de controle em contextos como trabalho, por exemplo.

\section{Referências}

Baltes, P. B. (1997). On the incomplete architecture of human ontogeny: Selection, optimization and compensation as foundation of developmental theory. American Psychologist, 52, 366-380.

Baltes, P. B., \& Baltes, M. M. (1990). Psychological perspectives on successful aging: The model of selective optimization with compensation. In P. B. Baltes, \& M. M. Baltes (Eds.), Successful aging: Perspectives from the behavioral sciences (pp. 1-34). New York: Cambridge University Press.

Brandtstädter, J., \& Renner, G. (1990). Tenacious goal pursuit and flexible goal adjustment: Explication and age-related analysis of assimilative and accommodative strategies of coping. Psychology and Aging, 5, 58-67.

Chipperfield, J. G., Newall, N. E., Perry, R. P., Stewart, T. L., Bailis, D. S., \& Ruthig, J. C. (2012). Sense of control in late life: Health and survival implications. Personality and Social Psychology Bulletin, 38, 1081-1092.

Chipperrfield, J. G., Perry, R. P., Bailis, D. S., Ruthig, J. C., \& Loring, P. C. (2007). Gender differences in use of primary and secondary control strategies in older adults with major health problems. Psychology \& Health, 22, 83-105.

Chiu, T., \& Spencer, G. A. (2010). Functional health status among rural and urban older adults in Taiwan: The effect of personal control and social control. International Journal of Older People Nursing, 5, 202-210.

Field, A. (2009). Descobrindo a estatística usando o SPSS. Porto Alegre: Artmed.

Haynes, T. L., Heckhausen, J., Chipperfield, J. G., Perry, R. P., \& Newall, N. E. (2009). Primary and secondary control strategies: Implications for health and well-being among older adults. Journal of Social and Clinical Psychology, 28, 165-197.

Heckhausen, J. (1997). Developmental regulation across adulthood: Primary and secondary control of age-related challenges. Developmental Psychology, 33, 176-87.

Heckhausen, J., \& Schulz, R. (1995). A life-span theory of control. Psychological Review, 102, 284-304.

Heckhausen, J., Schulz, R., \& Wrosch, C. (1998). Optimization in primary and secondary control: A measurement instrument for developmental regulation in adulthood [Technical Report]. Berlin: Max Planck Institute for Human Development.

Heckhausen, J., Wrosch, C., \& Fleeson, W. (2001). Developmental regulation before and after a developmental deadline: The sample case of "biological clock" for childbearing. Psychology and Aging, 16, 400-413.

Heckhausen, J., Wrosch, C., \& Schulz, R. (2010). A motivational theory of life-span development. Psychological Review, 117, 32-60.

Heeps, L. J. (2000). The role of primary/secondary control in positive psychological adjustment (Tese de doutorado não publicada). Deakin University, Austrália.

Khoury, H. T. T. (2005). Controle primário e controle secundário: relação com indicadores de envelhecimento bem-sucedido (Tese de Doutorado). Instituto de Psicologia. Universidade de Brasília. Brasília, DF. Retrieved from http://www.slideshare. net/Hilmapsi/tese-de-doutorado-percepo-de-controle-evelhice-bem-sucedida 
Khoury, H. T. T., \& Günther, I. A. (2009). Processos de autoregulação no curso de vida: controle primário e controle secundário. Psicologia: Reflexão e Crítica, 22, 144-152.

Lang, F. R., \& Heckhausen, J. (2001). Perceived control over development and subjective well-being: Differential benefits across adulthood. Journal of Personality and Social Psychology, 81, 509-523.

McConatha, J. T., \& Huba, H. M. (1999). Primary, secondary, and emotional control across adulthood. Current Psychology: Developmental, Learning, Personality, Social, 18, 164-170.

Ministério da Saúde (2006). Política Nacional de Saúde da Pessoa Idosa-PNSPI, Portaria No. 2528 de 19/10/2006. Brasília: MS.

Neri, A. L. (2006). Crenças de auto-eficácia e envelhecimento bem-sucedido. In E.V. Freitas, L. Py, F.A.X. Cançado, J. Doll, \& M.L. Gorzoni (Eds.), Tratado de geriatria e gerontologia ( $2^{\mathrm{a}}$ ed., pp. 1267-1276). Rio de Janeiro: Guanabara-Koogan.

Pasquali, L. (2005). Análise fatorial para pesquisadores. Brasília, DF: LabPAM. Pasquali, L. (2010). Testes referentes a construto: Teoria e modelo de construção. In L. Pasquali (Ed.), Instrumentação psicológica: fundamentos e práticas (pp. 165198). Porto Alegre: Artmed.

Peng, Y., \& Lachman, M. E. (1994, julho). Primary and secondary control: Cross cultural and life-span developmental perspectives. Paper presented at the 13th Biennial Meeting of Institutional Society for the Study of Behavioural Development. Amsterdan.

Rothbaum, F., Weisz, J. R., \& Snyder, S. S. (1982). Changing the world and changing the self: A two-process model of perceived control. Journal of Personality and Social Psychology, 42, 5-37.

Schulz, R., \& Heckhausen, J. (1996). A life-span model of successful aging. American Psychologist, 51, 702-714.

Seligman, M. E. P. (1977). Desamparo: Sobre depressão, desenvolvimento e morte. São Paulo: HUCITEC.

Stocks, A., April, K. A., \& Lynton, N. (2012). Locus of control and subjective well-being: A cross cultural study. Problems and perspectives in management, 10, 17-25.

Thurber, C. A., \& Weisz, J. R. (1997). "You can try or you can just give up": The impact of perceived control and coping style on childhood homesickness. Developmental Psychology, 33, 508-517.
Wahl, H. W., Schilling, O., \& Becker, S. (2007). Age-related macular degeneration and change in psychological control: Role of time since diagnosis and functional ability. Journal of Gerontology: Psychological Sciences, 62B (2), 90-97.

Weisz, J. R., Rothbaum, F. M., \& Blackburn, T. C. (1984). Standing out and standing in: The psychology of control in America and Japan. American Psychologist, 39, 955-969.

Widyarini, N. (2012). Control strategy as a model of psychological care for patient with diabetes. Health and the Environment Journal, 3(2), 86-91.

Windsor, T. D. (2009). Persistence in goal striving and positive reappraisal as psychological resources for ageing well: A dyadic analysis. Aging and Mental Health, 13, 874-884.

Wrosch, C., \& Heckhausen, J. (1999). Control processes before and after passing a developmental deadline: Activation and deactivation of intimate relationship goals. Journal of Personality and Social Psychology, 77, 415-427.

Wrosch, C., \& Heckhausen, J. (2002). Perceived control of life regrets: Good for young and bad for old adults. Psychology and Aging, 17, 340-350.

Wrosch, C., Heckhausen, J., \& Lachman, M. E. (2000). Primary and secondary control strategies for managing health and financial stress across adulthood. Psychology and Aging, 15, 387-399.

Wrosch, C., Schulz, R., \& Heckhausen, J. (2002). Health stress and depressive symptomatology in the elderly: The importance of health engagement control strategies. Health Psychology, 21, 340-348

Wrosch, C., Schulz, R., Miller, G. E., Lupien, S., \& Dunne, E. (2007). Physical health problems, depressive mood, and cortisol secretion in old age: Buffer effects of health engagement control strategies. Health Psychology, 26, 341-349.

Recebido em 28.03.2012

Primeira decisão editorial em 18.12.2012

Versão final em 06.03.2013

Aceito em 08.03.2013 